\title{
Avaliação físico-química e microbiológica de polpas de frutas congeladas comercializadas em Santarém-PA
}

\author{
Physicochemical and microbiological assessment of frozen fruit \\ pulps marketed in Santarém-PA
}

Yone Alves Sousa ${ }^{1}$, Mayara Aparecida Borges ${ }^{1}$, Alciene Ferreira da Silva Viana', Adrielle Leal Dias ${ }^{2}$, José Jeosafá Vieira de Sousa ${ }^{3}$, Bruno Alexandre da Silva ${ }^{1}$ (D, Silvia Katrine Rabelo da Silva ${ }^{3}$, Fagner Sousa de Aguiar ${ }^{1 *}$ (i)

${ }^{1}$ Universidade Federal do Oeste do Pará (UFOPA), Instituto de Saúde Coletiva, Santarém/PA - Brasil

2Universidade Federal do Oeste do Pará (UFOPA), Programa de Pós-graduação em Biociências, Santarém/PA - Brasil

${ }^{3}$ Universidade Federal do Oeste do Pará (UFOPA), Laboratório de Microbiologia (LabMicro), Santarém/PA - Brasil

${ }^{*}$ Corresponding Author: Fagner Sousa de Aguiar, Universidade Federal do Oeste do Pará (UFOPA), Avenida Mendonça Furtardo, 2946, Bairro Fátima, CEP: 68040-470, Santarém/PA - Brasil, e-mail: fagnersag@gmail.com

Cite as: Sousa, Y. A., Borges, M. A., Viana, A. F. S., Dias, A. L., Sousa, J. J. V., Silva, B. A., Silva, S. K. R. S., \& Aguiar, F. S. (2020). Physicochemical and microbiological assessment of frozen fruit pulps marketed in SantarémPA. Brazilian Journal of Food Technology, 23, e2018085. https://doi.org/10.1590/1981-6723.08518.

\begin{abstract}
Resumo
O presente estudo teve como objetivo a avaliação dos parâmetros físico-químicos e microbiológicos, e da atividade antioxidante de polpas de frutas produzidas e comercializadas no município de Santarém-PA. Foram analisadas três marcas de polpas congeladas de acerola (Malpighia emarginata), cupuaçu (Theobroma grandiflorum) e maracujá (Passiflora edulis), com a finalidade de verificar a sua adequação às normas e aos Padrões de Identidade e Qualidade (PIQ) estipulados pela legislação brasileira. Os parâmetros analisados foram $\mathrm{pH}$, acidez total titulável, sólidos solúveis totais, sólidos totais, cinzas e ácido ascórbico; buscou-se determinar o teor de polifenóis totais e da atividade antioxidante pelo método de DPPH, e foram realizadas análises microbiológicas para pesquisa de bolores e leveduras, coliformes fecais e Salmonella sp. As médias dos tratamentos foram comparadas pelo teste de Tukey, adotando-se o nível de significância $95 \%$. Os resultados revelaram que apenas o parâmetro pH estava de acordo com a legislação. Após a análise de acidez total titulável e sólidos totais, pôde-se constatar que todas as amostras não atenderam aos valores estabelecidos pela legislação. A análise de polifenóis totais e a atividade antioxidante revelaram que as polpas de acerola se destacaram pelo expressivo teor desses componentes, sendo estas consideradas como boa fonte de substâncias antioxidantes. Já os resultados das análises microbiológicas demonstraram que todas as amostras estavam de acordo com a legislação em vigor. As variações reportadas na maioria das análises mostram a necessidade da padronização dos processos produtivos das polpas.
\end{abstract}

Palavras-chave: Legislação; Qualidade; Cupuaçu; Maracujá; Acerola; Salmonella; Coliformes. 


\begin{abstract}
The present study aimed to evaluate the physical-chemical, microbiological parameters, and antioxidant activity of fruit pulps produced and marketed in Santarém-PA. Three manufacturers of frozen fruit pulp of acerola (Malpighia emarginata), cupuaçu (Theobroma grandiflorum), and passion fruit (Passiflora edulis) were analyzed, in order to verify their compliance to the Standards and Identity and Quality Standards (PIQ's) established by Brazilian legislation. The analyzed parameters were $\mathrm{pH}$, titratable total acidity, soluble solids, total solids, ash and ascorbic acid; determination of the total polyphenol content, and antioxidant activity by the DPPH method, and microbiological analyzes for mold and yeast, fecal coliforms and Salmonella sp. The means of the treatments were compared by the Tukey test, adopting the $95 \%$ level of significance. The results showed that only the $\mathrm{pH}$ parameter was in accordance with the legislation. After analysis of titratable total acidity and total solids, it was possible to verify that not all the samples met the values established by the legislation. The analysis of total polyphenols and the antioxidant activity revealed that the acerola pulp was featured by the expressive content of these components, being considered as a good source of antioxidant substances. The results of the microbiological analyzes showed that all the samples were in accordance with the legislation in force. The variations reported in the majority of the analyzes show the necessity of the standardization of the productive processes of the pulps.
\end{abstract}

Keywords: Legislation; Quality; Cupuassu; Passion Fruit; Acerola; Salmonella; Coliformes.

\title{
1 Introdução
}

As frutas apresentam grande importância na nutrição humana devido aos seus constituintes, como água, açúcares, fibras, vitaminas e sais minerais. Porém, devido à alta perecibilidade dos frutos e a problemas de armazenamento, que ocasionam perdas na produção, as polpas de frutas congeladas surgem como uma excelente alternativa de garantia de aproveitamento do excedente desses vegetais, podendo ser elaboradas nas épocas de safra e permitindo a oferta das polpas nos períodos de entressafra, evitando problemas ligados à sazonalidade (Castro et al., 2015).

Segundo a legislação brasileira do Ministério da Agricultura, polpa é o produto não fermentado, não concentrado ou diluído, obtido pelo esmagamento de frutos polposos. As frutas devem ser preparadas através de processos tecnológicos adequados que assegurem uma boa qualidade das suas características físico-químicas, nutricionais e microbiológicas, conforme estabelece na Instrução Normativa n. ${ }^{\circ} 49$, de 26 de setembro de 2018 (Brasil, 2018). Os compostos que apresentam atividade antioxidante não estão nos Padrões de Identidade de Qualidade (PIQ) de frutas no Brasil, entretanto são importantes por apresentarem efeitos biológicos positivos em humanos e animais (Pieniz et al., 2009).

As substâncias antioxidantes derivadas da dieta auxiliam o mecanismo de defesa no controle dos danos causados nas células pelos radicais livres. Dessa forma, é sabido que as substâncias antioxidantes são benéficas para o mecanismo de defesa celular, protegendo, assim, os componentes da célula de alterações oxidativas. A eficácia da ação antioxidante depende da estrutura química biodisponível e da concentração destes compostos (Pieniz et al., 2009; Leong et al., 2018).

A caracterização de parâmetros para controle de qualidade, bem como de compostos bioativos em polpas de frutas, agrega valor nutricional e assegura a qualidade do produto, visando atender não somente aos padrões estabelecidos pela legislação brasileira, mas também às exigências do consumidor.

Diante do exposto, este trabalho teve como objetivo avaliar os parâmetros físico-químicos e microbiológicos de três polpas de frutas produzidas e comercializadas no município de Santarém-PA. 


\section{Material e métodos}

\subsection{Amostragem}

Polpas de frutas congeladas (A, B, C), de três diferentes fabricantes, foram selecionadas em supermercados do município de Santarém-PA, sendo adquiridas três amostras de cada fabricante. As polpas de acerola, cupuaçu e maracujá foram selecionadas por serem as mais comercializadas, estando todas dentro do prazo de validade. As amostras foram transportadas em caixa de isopor com gelo para manter a integridade das mesmas, e acondicionadas em freezer $\left(-20^{\circ} \mathrm{C}\right)$. Após descongelamento a $4{ }^{\circ} \mathrm{C}$ e homogeneização das amostras, $100 \mathrm{~mL}$ foram utilizados para as determinações analíticas em triplicata.

\subsection{Análises físico-químicas}

\subsection{1 $\mathrm{pH}$}

$\mathrm{O} \mathrm{pH}$ foi determinado através do método potenciométrico, com um medidor digital de $\mathrm{pH}$, calibrado com tampões de pH 4 e 7, de acordo com o método 981.12 da Association of Official Analytical (2016).

\subsubsection{Acidez total titulável}

A acidez total titulável foi determinada por titulação com solução de $\mathrm{NaOH} 0,1 \mathrm{M}$, de acordo com o método 942.15 da Association of Official Analytical (2016), sendo os resultados expressos em gramas de ácido cítrico em 100 gramas de polpa (g ácido cítrico/100 g).

\subsubsection{Sólidos solúveis totais}

O teor de sólidos solúveis totais (SST) foi determinado por meio de leitura direta da amostra em refratômetro de Abbé a $20^{\circ} \mathrm{C}$, de acordo com o método 932.12 da Association of Official Analytical (2016), sendo os resultados expressos em ${ }^{\circ}$ Brix.

\subsubsection{Sólidos totais}

O teor de sólidos totais (ST) foi determinado pelo método gravimétrico, que se baseia na perda de peso das amostras submetidas a aquecimento à temperatura de $105^{\circ} \mathrm{C}$, em estufa, até peso constante, de acordo com o método 934.06 da Association of Official Analytical (2016).

\subsubsection{Cinzas}

As análises de cinzas foram determinadas utilizando o método gravimétrico, que se baseia na calcinação da matéria orgânica em forno mufla a $550{ }^{\circ} \mathrm{C}$, de acordo com o método 940.26 da Association of Official Analytical (2016).

\subsection{6 Ácido ascórbico}

O teor de ácido ascórbico foi determinado através do método titulométrico, baseado na redução do indicador 2,6-diclorofenolindofenol, de acordo com o método 967.21 da Association of Official Analytical (1999). 


\subsection{Polifenóis totais}

O conteúdo de compostos fenólicos foi determinado com base no método colorimétrico de Folin-Ciocalteau, segundo Singleton \& Rossi (1965), em espectrofotômetro UV-VIS a $740 \mathrm{~nm}$, sendo os resultados expressos em miligrama equivalente de ácido gálico em 100 gramas de polpa (mg eq. ácido gálico/100 g).

\subsection{Atividade antioxidante}

A atividade antioxidante foi feita pelo método químico de sequestro do radical livre DPPH, conforme descrito por Silva et al. (2012a), com algumas modificações. Foram realizadas em cinco concentrações diferentes, em que a soma da amostra e do metanol resultou em um volume de 600 microlitros, adicionados em tubos de ensaio contendo 2,4 mL de solução metanólica de DPPH 0,06 mM. A diminuição da absorbância a $516 \mathrm{~nm}$ foi medida em intervalos de 40 minutos até estabilização da reação. O percentual de inibição dos radicais DPPH para cada amostra foi calculada de acordo com a equação 1:

$$
I D P P H(\%)=100 X(A b s B-A b s A) / A b s B
$$

Em que: $A b s A$ e $A b s B$ são as absorbâncias da amostra e do controle (branco) no término da reação, respectivamente.

A atividade do sequestro do DPPH foi expressa por meio da concentração mínima efetiva para reduzir em $50 \%$ a concentração inicial do radical. Os valores de IC $50 \% \mathrm{em} \mathrm{mg} / \mathrm{mL}$ foram obtidos por modelos adequados de regressão $(p<0,05)$ utilizando os valores de concentração das amostras versus o percentual de inibição $\left(\mathrm{r}^{2}>0,95\right)$.

\subsection{Análises microbiológicas}

As análises microbiológicas foram realizadas no Laboratório de Microbiologia da Universidade Federal do Oeste do Pará - UFOPA. Foram pesados 25 gramas de cada amostra e transferidos para frascos de diluição contendo $225 \mathrm{~mL}$ de água peptonada estéril (diluição $10^{-1}$ ) e, a partir dessa diluição, foram feitas as diluições seguintes até $10^{-3}$. A determinação de bolores e leveduras, Salmonella sp. e a de coliformes a $45{ }^{\circ} \mathrm{C}$ foi realizada segundo a metodologia de Silva et al. (2007), utilizando o método de plaqueamento direto em superfície das diluições seriadas.

\subsection{Análise estatística}

O tratamento estatístico foi realizado com o auxílio do programa STATISTICA versão 7.1 sob a plataforma Windows $10^{\circledR}$. As médias dos tratamentos foram comparadas pelo teste de Tukey $(\alpha=0,05)$.

\section{Resultados e discussão}

De acordo com os resultados físico-químicos obtidos (Tabela 1), o $\mathrm{pH}$ das polpas de acerola variou de 3,26 a 3,60, sendo que o fabricante A foi o que apresentou o maior valor. Para as polpas de cupuaçu e maracujá, os valores variaram de 3,27 a 3,54 e 2,80 a 3,31, respectivamente, havendo diferença estatística entre todas as marcas $(p<0,05)$. Entre as polpas de cupuaçu e maracujá, o fabricante $\mathrm{C}$ apresentou os menores valores de pH: 3,27 e 2,80, respectivamente. Já o fabricante A apresentou os maiores valores de $\mathrm{pH}, 3,54$ e 3,31 para as respectivas polpas.

Estudos realizados por Urbano et al. (2012) obtiveram um valor médio de pH de 3,25 e Caldas et al. (2010) relataram uma média de 3,38 $\pm 0,15$ para polpa de acerola. Para a polpa de cupuaçu, Canuto et al. (2010) relataram em seu trabalho a média de 3,5 $\pm 0,2$. Freire et al. (2009), avaliando as caraterísticas físico-químicas de polpas congeladas de cupuaçu, encontraram um valor de 3,45 $\pm 0,01$. Raimundo et al. (2009) encontraram os valores médios de 2,92 a 3,1, e Santos et al. (2016) encontraram um valor médio de 3,33 para polpas de maracujá. 
Tabela 1. Média e desvio padrão dos parâmetros físico-químicos de polpas de frutas congeladas, produzidas e comercializas no município de Santarém-PA.

\begin{tabular}{|c|c|c|c|c|c|}
\hline Amostras & pH & $\begin{array}{c}\text { ATT } \\
(\mathrm{g} / 100 \mathrm{~g})\end{array}$ & $\begin{array}{c}\text { SS } \\
\left({ }^{(} \text {Brix }\right) \\
\end{array}$ & $\begin{array}{c}\text { ST } \\
(\mathrm{g} / \mathbf{1 0 0} \mathrm{g})\end{array}$ & $\begin{array}{c}\text { Cinzas } \\
(\mathrm{g} / 100 \mathrm{~g})\end{array}$ \\
\hline \multicolumn{6}{|l|}{ Acerola } \\
\hline $\mathrm{A}$ & $3,60 \pm 0,05^{\mathrm{a}}$ & $0,22 \pm 0,0^{\mathrm{a}}$ & $5,24 \pm 0,01^{\mathrm{a}}$ & $5,77 \pm 0,17^{\mathrm{a}}$ & $0,20 \pm 0,01^{\mathrm{a}}$ \\
\hline $\mathrm{B}$ & $3,26 \pm 0,02^{b}$ & $0,25 \pm 0,01^{\mathrm{b}}$ & $10,24 \pm 0,01^{\mathrm{b}}$ & $5,91 \pm 0,04^{\mathrm{a}}$ & $0,24 \pm 0,00^{\mathrm{b}}$ \\
\hline $\mathrm{C}$ & $3,29 \pm 0,01^{b}$ & $0,25 \pm 0,01^{\mathrm{b}}$ & $10,24 \pm 0,01^{\mathrm{b}}$ & $5,48 \pm 0,09^{b}$ & $0,25 \pm 0,01^{\mathrm{b}}$ \\
\hline \multicolumn{6}{|l|}{ PIQ } \\
\hline Mín & 2,80 & 0,80 & 5,5 & 6,50 & - \\
\hline Máx & - & - & - & - & - \\
\hline \multicolumn{6}{|l|}{ Cupuaçu } \\
\hline $\mathrm{A}$ & $3,54 \pm 0,01^{\mathrm{a}}$ & $0,47 \pm 0,02^{\mathrm{a}}$ & $6,24 \pm 0,01^{\mathrm{a}}$ & $7,05 \pm 0,45^{\mathrm{a}}$ & $0,35 \pm 0,03^{\mathrm{ab}}$ \\
\hline $\mathrm{B}$ & $3,46 \pm 0,01^{\mathrm{b}}$ & $0,56 \pm 0,00^{\mathrm{b}}$ & $7,98 \pm 0,01^{\mathrm{b}}$ & $9,06 \pm 0,02^{b}$ & $0,32 \pm 0,02^{b}$ \\
\hline $\mathrm{C}$ & $3,27 \pm 0,03^{c}$ & $0,46 \pm 0,01^{\mathrm{a}}$ & $7,98 \pm 0,01^{\mathrm{b}}$ & $7,66 \pm 0,22^{\mathrm{a}}$ & $0,28 \pm 0,02^{\mathrm{cb}}$ \\
\hline \multicolumn{6}{|l|}{ PIQ } \\
\hline Mín & 2,60 & & 9,0 & 12,0 & - \\
\hline Máx & - & 1,50 & - & - & - \\
\hline \multicolumn{6}{|l|}{ Maracujá } \\
\hline $\mathrm{A}$ & $3,31 \pm 0,02^{\mathrm{a}}$ & $0,16 \pm 0,01^{\mathrm{a}}$ & $5,74 \pm 0,01^{\mathrm{a}}$ & $5,45 \pm 0,25^{\mathrm{a}}$ & $0,29 \pm 0,00^{\mathrm{a}}$ \\
\hline $\mathrm{B}$ & $2,92 \pm 0,02^{b}$ & $0,44 \pm 0,02^{\mathrm{b}}$ & $15,03 \pm 0,64^{b}$ & $10,64 \pm 0,28^{b}$ & $0,53 \pm 0,02^{b}$ \\
\hline $\mathrm{C}$ & $2,80 \pm 0,02^{\mathrm{c}}$ & $0,43 \pm 0,01^{\mathrm{b}}$ & $12,93 \pm 0,06^{\mathrm{c}}$ & $9,14 \pm 0,09^{c}$ & $0,33 \pm 0,02^{\mathrm{a}}$ \\
\hline \multicolumn{6}{|l|}{ PIQ } \\
\hline Mín & 2,7 & 2,50 & 11,0 & 11,0 & - \\
\hline Máx & - & - & - & - & - \\
\hline
\end{tabular}

ATT: Acidez Total Titulável; SS: Sólidos Solúveis; ST: Sólidos Totais. A, B e C são os diferentes fabricantes. PIQ: Padrão de Indetidade e Qualidade. Médias seguidas por letras iguais não diferem pelo teste de Tukey $(\alpha=0,05)$.

Todas as polpas de acerola e cupuaçu apresentaram valores acima do padrão mínimo estabelecido para o pH, porém a legislação não preconiza um valor máximo para essas polpas de frutas. As polpas de maracujá apresentaram-se em conformidade com os padrões exigidos pela legislação brasileira, em que a faixa mínima é de 2,7 (Brasil, 2018). Segundo Lira Júnior et al. (2005), o pH é estabelecido como atributo de qualidade do produto pela legislação por favorecer a conservação da polpa, evitando o crescimento de microrganismos.

$\mathrm{Na}$ análise da acidez total titulável, os valores variaram de $0,22 \%$ a $0,25 \%$ para as polpas de acerola, sendo que o fabricante $\mathrm{A}$ apresentou o menor valor $(0,22 \%)$. Para as polpas de cupuaçu, os valores variaram de $0,46 \%$ a $0,56 \%$, sendo que o fabricante B apresentou o maior valor $(0,56 \%)$. Entre as polpas de maracujá, os valores variaram de $0,16 \%$ a $0,44 \%$, sendo que o fabricante $A$ apresentou o menor valor $(0,16 \%)$.

Todas as amostras analisadas encontraram-se em desacordo com os padrões preconizados pela legislação, estando os resultados inferiores aos que são exigidos. De acordo com o PIQ, para polpa de acerola, a acidez mínima deve ser de $0,80 \%$; para cupuaçu, deve ser de $1,50 \%$, e para o maracujá, deve ser de $2,50 \%$. Estes resultados podem ser atribuídos ao fato de a acidez estar correlacionada diretamente a fatores externos, tais como clima, solo, tempo de maturação da fruta, ou por problemas durante o processamento, como adição excessiva de água (Leal et al., 2013).

O teor de sólidos solúveis para as polpas de acerola e cupuaçu variaram de 5,24 a $10,24{ }^{\circ} \mathrm{B}$, e 6,24 a $7,98{ }^{\circ} \mathrm{B}$, respectivamente, sendo que o fabricante $\mathrm{A}$ apresentou os menores valores $\left(5,24^{\circ} \mathrm{B}\right)$ e $\left(6,24{ }^{\circ} \mathrm{B}\right)$ para ambas as polpas. Com relação às polpas de maracujá, todas as marcas diferiram estatisticamente $(p<0,05)$, sendo que o fabricante $\mathrm{A}$ apresentou o menor valor $\left(5,74^{\circ} \mathrm{B}\right)$ e o fabricante $\mathrm{B}$ apresentou o maior valor $\left(15,4^{\circ} \mathrm{B}\right)$.

Para a polpa de acerola, apenas o fabricante A apresentou valor inferior ao estabelecido pelo PIQ, que é 5,5 $\mathrm{B}$. No trabalho de Caldas et al. (2010), das sete marcas avaliadas, duas apresentaram valores em desacordo com a legislação em relação aos sólidos solúveis, sendo obtidos os resultados $5,16^{\circ} \mathrm{B}$ e $5,13^{\circ} \mathrm{B}$, inferiores aos encontrados neste trabalho. Para o sabor cupuaçu, todas as marcas apresentaram resultados inferiores, em que a legislação estabelece o valor mínimo de $9,0^{\circ} \mathrm{B}$. Para a polpa de maracujá, apenas o fabricante A apresentou resultado inferior, 


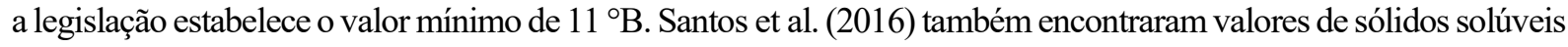
inferiores ao mínimo estabelecido pela legislação vigente $\left(8,20^{\circ} \mathrm{B}\right.$ e $\left.6,50^{\circ} \mathrm{B}\right)$ nas polpas de maracujá.

Diversos fatores, como clima, pluviosidade durante o cultivo e adição de água durante o processo de fabricação, podem ter efeito sobre o teor de sólidos solúveis nas polpas, o que justificaria a falta de uniformidade entre os valores apresentados para as diferentes marcas (Freire et al., 2009). O baixo teor de sólidos solúveis também pode ser causado por processamento inadequado e baixa qualidade da matéria-prima (Brasil, 2000).

$\mathrm{Na}$ análise estatística de sólidos totais das polpas de acerola, os valores variaram de 5,48\% a 5,91\%, sendo que o fabricante $\mathrm{C}$ apresentou o menor valor $(5,48 \%)$. Nas polpas de cupuaçu, os valores variaram de 7,05\% a 9,06\%, em que o fabricante B apresentou o maior valor (9,06\%). Com relação às polpas de maracujá, todas as marcas diferiram estatisticamente $(p<0,05)$, sendo que o fabricante A apresentou o menor valor $(5,45 \%)$ e o fabricante B apresentou o maior valor $(10,64 \%)$.

Em outros trabalhos com polpas de frutas congeladas, valores abaixo do que preconiza o PIQ também foram encontrados. Paglarini et al. (2011) obtiveram médias de 5,57\% e 5,42\% para polpas de acerola, com variação entre $8,61 \pm 0,26 \%$ e $10,11 \pm 1,37 \%$ para polpas de cupuaçu e, para polpa de maracujá, valores entre $9,34 \pm 0,26 \%$ e $10,24 \pm 0,09 \%$. Moisés et al. (2016) encontraram médias entre $6,19 \%$ e $9,87 \%$ para cupuaçu e maracujá, respectivamente. Essa análise tem, principalmente, a finalidade de verificar adição de água durante o processamento das polpas, o que é proibido pela legislação brasileira para este tipo de produto (Brasil, 2018).

O conteúdo de cinzas das amostras de acerola variou de $0,20 \%$ a $0,25 \%$, e de maracujá, variou de $0,29 \%$ a $0,53 \%$. No estudo realizado por Lima et al. (2015), foram encontrados os valores $0,19 \%$ e $0,25 \%$ para as respectivas polpas. Para as amostras de cupuaçu os valores variaram de $0,28 \%$ a $0,35 \%$, sendo estes resultados inferiores ao encontrado por Gonçalves et al. (2013), que obtiveram a média de $0,83 \%$. Este parâmetro é fortemente influenciado por fatores edafoclimáticos, como o índice pluviométrico e as condições do solo; portanto, a grande variação é esperada.

Os valores encontrados nas análises de quantificação de ácido ascórbico, polifenóis totais e capacidade antioxidante estão apresentados na Tabela 2.

Tabela 2. Teores de ácido ascórbico, polifenóis totais e capacidade antioxidante das polpas congeladas.

\begin{tabular}{cccc}
\hline AMOSTRAS & AA $(\mathbf{g} / \mathbf{1 0 0} \mathbf{g})$ & PT $(\mathbf{m g}$ EAG/100 g) & IC $_{\mathbf{5 0}}(\mathbf{m g} / \mathbf{m l})$ \\
\hline Acerola & & & \\
\hline A & $1112 \pm 25^{\mathrm{a}}$ & $1005,2 \pm 19^{\mathrm{a}}$ & $19,6 \pm 3,2^{\mathrm{a}}$ \\
\hline B & $1437 \pm 11^{\mathrm{b}}$ & $843,8 \pm 16^{\mathrm{b}}$ & $16,6 \pm 0,0^{\mathrm{a}}$ \\
\hline C & $1615 \pm 10^{\mathrm{b}}$ & $1069,5 \pm 64^{\mathrm{a}}$ & $8,6 \pm 0,0^{\mathrm{b}}$ \\
\hline Cupuaçu & & & $70,5 \pm 3,7^{\mathrm{a}}$ \\
\hline A & $116 \pm 25^{\mathrm{a}}$ & $32,5 \pm 1,1^{\mathrm{a}}$ & $106,6 \pm 2,9^{\mathrm{b}}$ \\
\hline B & $120 \pm 2^{\mathrm{a}}$ & $31,9 \pm 1,1^{\mathrm{a}}$ & $92,8 \pm 3,0^{\mathrm{c}}$ \\
\hline C & $174 \pm 0^{\mathrm{b}}$ & $27,9 \pm 1,1^{\mathrm{b}}$ & $110,5 \pm 2,9^{\mathrm{a}}$ \\
\hline Maracujá & & & $57,3 \pm 3,6^{\mathrm{b}}$ \\
\hline A & $132 \pm 0^{\mathrm{a}}$ & $791,5 \pm 5,0^{\mathrm{a}}$ & $122,6 \pm 3,0^{\mathrm{c}}$ \\
\hline B & $174 \pm 2^{\mathrm{b}}$ & $662,9 \pm 9,0^{\mathrm{b}}$ & \\
\hline C & $210 \pm 22^{\mathrm{c}}$ & $34,0 \pm 5,0^{\mathrm{c}}$ & \\
\hline
\end{tabular}

AA: Ácido Ascórbico; PT: Polifenois Totais; EAG: Equivalente Ácido Gálico; $\mathrm{IC}_{50}$ (concentração mínima efetiva para reduzir em $50 \%$ a concentração inicial do radical DPPH): TROLOX 3,06 $\mu \mathrm{g} / \mathrm{mL}$. A, B e C são os diferentes fabricantes. Médias seguidas por letras iguais não diferem pelo teste de Tukey $(\alpha=0,05)$.

Quanto ao parâmetro de ácido ascórbico, a legislação estabelece valores apenas para as polpas de acerola e cupuaçu. Analisando a Tabela 2, observa-se que a acerola apresentou os maiores valores de vitamina $\mathrm{C}$ em comparação com as outras polpas $(1.112 \pm 25 \mathrm{mg} / 100 \mathrm{~g}$ a $1.615 \pm 108 \mathrm{mg} / 100 \mathrm{~g})$, sendo que a legislação estabelece o valor mínimo de $800 \mathrm{mg} / 100 \mathrm{~g}$. Esses resultados são superiores aos encontrados nos estudos 
realizados por Caldas et al. (2010) e Brasil et al. (2016), que obtiveram um valor médio de 124,12 mg/100 g e 593,14 mg/100 g, respectivamente. Já Bueno et al. (2002) e Sebastiany et al. (2009) encontraram os valores de $1.374,2 \mathrm{mg} / 100 \mathrm{~g}$ e $1.853,37 \mathrm{mg} / 100 \mathrm{~g}$, respectivamente, na polpa de acerola.

Nas polpas de cupuaçu, foram encontrados valores entre $116 \pm 25 \mathrm{mg} / 100 \mathrm{~g}$ e $174 \pm 0 \mathrm{mg} / 100 \mathrm{~g}$. De acordo com a legislação, o valor mínimo para cupuaçu é de $18 \mathrm{mg} / 100 \mathrm{~g}$. Em estudo realizado por Nascimento et al. (2012), obteve-se um valor médio de $84,50 \mathrm{mg} / 100 \mathrm{~g}$ de polpa, resultado inferior ao encontrado neste trabalho.

Entre as polpas de maracujá, todas as marcas apresentaram diferença estatística significativa $(p<0,05)$, sendo que o fabricante $\mathrm{C}$ apresentou o maior valor $(210,3 \pm 22 \mathrm{mg} / 100 \mathrm{~g})$ e o fabricante A apresentou o menor valor $(132 \pm 0 \mathrm{mg} / 100 \mathrm{~g})$. Apesar de a legislação não estabelecer um valor de referência para o parâmetro ácido ascórbico em polpa de maracujá, ao comparar com os resultados encontrados por Costa (2016) $(15,54 \pm 0,61 \mathrm{mg} / 100 \mathrm{~g})$ e Lima et al. (2015) $(6,21 \pm 0,05 \mathrm{mg} / 100 \mathrm{~g})$, observa-se que os valores encontrados neste estudo são bastante superiores.

$\mathrm{Na}$ análise de polifenóis totais, para as polpas de acerola, os valores variaram de $843,8 \pm 16 \mathrm{mg}$ EAG/100 g a $1.069,5 \pm 64 \mathrm{mg}$ EAG/100 g, sendo que o fabricante B apresentou o menor valor (843,8 $\pm 16 \mathrm{mg}$ EAG/100 g). Entre

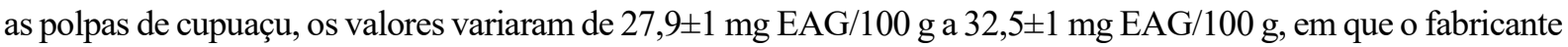
$\mathrm{C}$ apresentou o menor valor $(27,9 \pm 1,1 \mathrm{mg}$ EAG/100 g). Para as polpas de maracujá, todas as marcas apresentaram diferença estatística significativa $(p<0,05)$, sendo que o fabricante $\mathrm{C}$ apresentou o menor valor ( $34 \pm 5 \mathrm{mg} \mathrm{EAG} / 100 \mathrm{~g}$ ) e o fabricante A apresentou o maior valor (791,5 $\pm 5 \mathrm{mg} \mathrm{EAG} / 100 \mathrm{~g}$ ).

As polpas de acerola e maracujá, com exceção do fabricante $\mathrm{C}$, apresentaram quantidades consideráveis de polifenóis totais, com ênfase para as polpas de acerola, que exibiram maiores teores destes compostos. Kuskoski et al. (2006) relataram valores de 580,1 $\pm 4,6,20,5 \pm 3$ e $20 \pm 2,6 \mathrm{mg}$ EAG/100 g para as polpas de acerola, cupuaçu e maracujá, respectivamente. Dessa forma, os teores de polifenóis totais das polpas utilizadas neste estudo são superiores aos relatados por esses autores.

Os valores de $\mathrm{IC}_{50}$ das polpas de acerola variaram de $8,6 \pm 0$ a 19,6 $2 \pm 3,2 \mathrm{mg} / \mathrm{mL}$. Esperavam-se estes valores menores dentre as polpas estudadas, por apresentarem alto teor de ácido ascórbico. Quanto às polpas de cupuaçu e maracujá, os valores variaram de 70,5 $\pm 3,7$ a $106,6 \pm 2,9 \mathrm{mg} / \mathrm{mL}$ e $57,3 \pm 3,6$ a $122,6 \pm 3 \mathrm{mg} / \mathrm{mL}$, respectivamente, sendo observado que todas as marcas apresentaram diferença estatística significativa $(p<0,05)$.

Entre as polpas de acerola, o fabricante $\mathrm{C}$ foi o que apresentou menor valor de $\mathrm{IC}_{50}(8,6 \pm 0 \mathrm{mg} / \mathrm{mL})$; entre as polpas de cupuaçu, o fabricante $A$ apresentou o menor valor de $\mathrm{IC}_{50}(70,5 \pm 3,7 \mathrm{mg} / \mathrm{mL})$, e entre as polpas de maracujá, o fabricante $B$ foi o que apresentou menor valor $(57,3 \pm 3,6 \mathrm{mg} / \mathrm{mL})$, o que nos faz inferir que estas marcas de polpas citadas possuem a maior capacidade antioxidante em relação às demais polpas. Segundo Kuskoski et al. (2005), a atividade antioxidante é conferida às frutas por meio de seus compostos antioxidantes e os maiores responsáveis por essa funcionalidade são os compostos fenólicos e vitaminas. Assim, o fato de o fabricante $\mathrm{C}$ ter apresentado o maior valor de compostos fenólicos e ácido ascórbico para as polpas de acerola e o fabricante A, para as polpas de cupuaçu, justifica a maior capacidade antioxidante destas.

De acordo com as análises para a determinação de coliformes a $45^{\circ} \mathrm{C}$ e Salmonella sp., todas as polpas não apresentaram crescimento, estando de acordo com a Resolução RDC n. ${ }^{\circ} 12$, de 02 de janeiro de 2001 - ANVISA (Tabela 3). A ausência da contagem de microrganismos dos grupos de coliformes e de Salmonella sp., nas polpas de frutas, está associada à qualidade satisfatória do produto, que se dá, possivelmente, por meio de adequada aplicação das técnicas de processamento e/ou armazenamento (Castro et al., 2015). Além disso, o baixo valor de $\mathrm{pH}$ das polpas de frutas inibe o crescimento de Salmonella sp. Outros autores também não observaram o crescimento desses microrganismos em pesquisas realizadas com polpas de frutas, como Sebastiany et al. (2009), Araújo (2015) e Urbano et al. (2012). 
Tabela 3. Contagem de bolores e leveduras, coliformes a $45{ }^{\circ} \mathrm{C}$ e Salmonella $s p$.

\begin{tabular}{|c|c|c|c|}
\hline AMOSTRA & $\begin{array}{c}\text { Bolores e Leveduras } \\
\text { (UFC/g) }\end{array}$ & $\begin{array}{c}\text { Coliformes a } 45^{\circ} \mathrm{C} \\
\text { (UFC/g) }\end{array}$ & $\begin{array}{c}\text { Salmonella sp. } \\
(\mathrm{em} 25 \mathrm{~g})\end{array}$ \\
\hline \multicolumn{4}{|l|}{ Acerola } \\
\hline $\mathbf{A}$ & $1,0 \times 10^{2}$ & $<1,0 \times 10^{1}$ & Ausente \\
\hline B & $4,0 \times 10^{1}$ & $<1,0 \times 10^{1}$ & Ausente \\
\hline $\mathrm{C}$ & $4,0 \times 10^{1}$ & $<1,0 \times 10^{1}$ & Ausente \\
\hline \multicolumn{4}{|l|}{ Cupuaçu } \\
\hline A & $3,0 \times 10^{1}$ & $<1,0 \times 10^{1}$ & Ausente \\
\hline B & $3,0 \times 10^{1}$ & $<1,0 \times 10^{1}$ & Ausente \\
\hline $\mathrm{C}$ & $4,0 \times 10^{1}$ & $<1,0 \times 10^{1}$ & Ausente \\
\hline \multicolumn{4}{|l|}{ Maracujá } \\
\hline $\mathbf{A}$ & $1,1 \times 10^{2}$ & $<1,0 \times 10^{1}$ & Ausente \\
\hline B & $3,4 \times 10^{2}$ & $<1,0 \times 10^{1}$ & Ausente \\
\hline $\mathbf{C}$ & $6,0 \times 10^{1}$ & $<1,0 \times 10^{1}$ & Ausente \\
\hline
\end{tabular}

UFC: Unidades Formadoras de Colônia; A, B e C são os diferentes fabricantes. Médias seguidas por letras iguais não diferem pelo teste de Tukey $(\alpha=0,05)$.

A ANVISA não estabelece padrões para bolores e leveduras em polpas de frutas, contudo os resultados das análises para estes microrganismos foram avaliados de acordo com a Instrução Normativa $\mathrm{n}^{\circ} 49$, de 26 de setembro de 2018, que estabelece que a soma de bolores e leveduras seja, no máximo, $5 \times 10^{3} / \mathrm{g}$ para polpa in natura congelada ou não (Brasil, 2018). De acordo com os valores encontrados neste trabalho, todas as amostras apresentam-se dentro dos padrões exigidos pela legislação. Em alimentos congelados, espera-se encontrar baixas contagens de fungos filamentosos e leveduras, o que pode ser justificado pelo emprego de temperatura ideal na conservação das polpas e pela adequada condição higiênica.

Castro et al. (2015) apresentaram resultados semelhantes, para todos os microrganismos, aos encontrados no presente estudo. Silva et al. (2012b) também obtiveram resultados semelhantes ao analisarem polpas de acerola pasteurizadas e congeladas.

\section{Conclusão}

Após as análises físico-químicas, constatou-se que há a necessidade de adequação da maioria dos parâmetros para atendimento dos Padrões de Identidade e Qualidade (PIQ) vigentes e estipulados pela legislação brasileira. As variações encontradas nos resultados são indícios de falhas no processo produtivo das polpas de frutas produzidas e comercializadas no munícipio de Santarém-PA. Todos os fabricantes avaliados continham compostos fenólicos, com ênfase para as polpas de acerola e maracujá, exceto o fabricante $\mathrm{C}$ deste último sabor. Os resultados da atividade antioxidante revelaram que as polpas de acerola podem ser consideradas como boas fontes de substâncias antioxidantes. Os resultados das análises microbiológicas demonstram que todas as amostras estavam de acordo com a legislação em vigor.

\section{Referências}

Araújo, R. P. S. (2015). Avaliação da qualidade microbiológica de polpas de frutas comercializadas no município de Currais Novos/RN (Trabalho de Conclusão de Curso). Recuperado em 17 de janeiro de 2018, de https://memoria.ifrn.edu.br/handle/1044/828

Association of Official Analytical - AOAC. (1999). Official methods of analysis of the Association of Official Analytical Chemists (15th ed.) Arlington: AOAC.

Association of Official Analytical - AOAC. (2016). Official methods of analysis of the Association of Official Analytical Chemists (20th ed.) Gaithersburg: AOAC.

Brasil, A. S., Sigarini, K. S., Pardinho, F. C., Faria, R. A. P. G., \& Siqueira, N. F. M. P. S. (2016). Avaliação da qualidade físicoquímica de polpas de fruta congeladas comercializadas na cidade de Cuiabá - MT. Revista Brasileira de Fruticultura, 38(1), 167-175. http://dx.doi.org/10.1590/0100-2945-253/14 
Brasil. Ministério da Agricultura e do Abastecimento (2000, janeiro 7). Regulamento técnico geral para fixação dos padrões de identidade e qualidade para polpa de frutas (Instrução Normativa $n^{\circ} 1$ ). Diário Oficial [da] União, Brasília.

Brasil. Ministério da Agricultura e do Abastecimento (2018, setembro 26). Resolve: Fica estabelecida em todo o território nacional a complementação dos Padrões de Identidade e Qualidade de Suco e Polpa de Fruta, na forma desta Instrução Normativa (Instrução Normativa $n^{\circ}$ 49). Diário Oficial [da] União, Brasília.

Bueno, S. M., Lopes, M. R. V., Graciano, R. A. S., Fernandes, E. C. B., \& Garcia-Cruz, C. H. (2002). Avaliação da qualidade de Polpas de Frutas Congeladas. Revista do Instituto Adolfo Lutz, 62(2), 121-126.

Caldas, Z. T. C., Araújo, F. M. M. C., Machado, A. V., Almeida, A. K. L., \& Alves, F. M. S. (2010). Investigação de qualidade das polpas de frutas congeladas comercializadas nos estados da Paraíba e Rio Grande do Norte. Revista Verde de Agroecologia e Desenvolvimento Sustentável, 5(4), 156-163.

Canuto, G. A. B., Xavier, A. A. O., Leandro, C. N. \& De Benassi, M. T. (2010). Caracterização físico-química de polpas de frutos da amazônia e sua correlação com a atividade anti-radical livre. Revista Brasileira de Fruticultura, 32(4), 1196-1205.

Castro, T. M. N., Zamboni, P. V., Dovadoni, S., Cunha Neto, A., \& Rodrigues, L. J. (2015). Parâmetros de qualidade de polpas de frutas congeladas. Revista do Instituto Adolfo Lutz, 74(4), 426-436.

Costa, J. O (2016). Determinação do teor de vitamina C em polpas de frutas congeladas por lodimetria: uma opção para o controle de qualidade? (Trabalho de Conclusão de Curso). Recuperado em19 de dezembro de 2017, de https://repositorio.ufpe.br/handle/123456789/23878?locale=pt_BR

Freire, M. T. A., Petrus, R. R., Freire, C. M. A., Oliveira, C. A. F., Felipe, A. M. P. F., \& Gatti, J. B. (2009). Caracterização físicoquímica, microbiológica e sensorial de polpa de cupuaçu congelada (Theobroma grandiflorum Shum). Brazilian Journal of Food Technology, 12(1), 9-16. http://dx.doi.org/10.4260/BJFT2009060800002

Gonçalves, M. V. V. A., Silva, J. P. L., Mathias, S. P., Rosenthal, A., Calado, V. M. A. (2013). Caracterização físico-química e reológicas da polpa de cupuaçu congelada (Theobroma grandiflorum Schum.). Perspectivas Online: Exatas \& Engenharia, 3(7), 46-53.

Kuskoski, E. M., Asuero, A. G., Morales, M. T., \& Fett, R. (2006). Frutos tropicais silvestres e polpas de frutas congeladas: atividade antioxidante, polifenóis e antocianinas. Ciência Rural, 36(4), 1283-1287. http://dx.doi.org/10.1590/S010384782006000400037

Kuskoski, E. M., Asuero, A. G., Trancoso, A. M., Mancini-Filho, J., Fett, R. (2005). Aplicación de diversos métodos químicos para determinar actividad antioxidante en pulpa de frutos. Ciência e Tecnologia dos Alimentos, 24(4), 726-732.

Leal, R. C., Reis, V. B., \& Luz, D. A. (2013). Avaliação de parâmetros físico-químicos de polpas congeladas de graviola comercializada em supermercados de São Luís - MA. Cadernos de Pesquisa, 20(2), 76-80. http://dx.doi.org/10.18764/21782229.v20n2p76-80

Leong, H. Y., Show, P. L., Lim, M. H., Ooi, C. W., \& Ling, T. C. (2018). Natural red pigments from plants and their health benefits: A review. Food Reviews International, 35(5), 463-482. http://dx.doi.org/10.1080/87559129.2017.1326935

Lima, T. L. S., Cavalcante, C. L., Sousa, D. G., Silva, P. H. A., \& Andrade Sobrinho, L. G. (2015). Avaliação da composição físico-química de polpas de frutas comercializadas em cinco cidades do Alto Sertão paraibano. Revista Verde, 10(2), 49-55. http://dx.doi.org/10.18378/rvads.v10i2.3378

Lira Júnior, J. S., Musser, R. S., Melo, E. A., Maciel, M. I. S., Lederman, I. E., \& Santos, V. F. (2005). Caracterização física e físico-química de frutos de cajá-umbu (Spondias spp.). Food Science and Technology, 25(4), 757-761. http://dx.doi.org/10.1590/S0101-20612005000400021

Moisés, R. M. M., Mendes, A. H. L., Aquino, C. M., Moreira, L. F., Barbosa, M. C. F. \& Moura, F. J. (2016). Avaliação fisicoquímica de polpas de frutas congeladas produzidas em um município do interior do Ceará. In SBCTA (Ed.), XXV Congresso Brasileiro de Ciência e Tecnologia de Alimentos (pp. 1-6). Gramado, RS: SBCTA.

Nascimento, C. R., Neves, L. C., Grígio, M. L., Campos, A. J., Chagas, E. A., \& Souza, A. A. (2012). Avaliação da qualidade de polpas de frutos industrializados e comercializados no município de Boa Vista - RR. Revista. Agroambiente On-Line, 6(3), 263267. http://dx.doi.org/10.18227/1982-8470ragro.v6i3.680

Paglarini, C. S., Silva, F. S., Porto, A. G., Santos, P. \& Leite, A. L. M. P. (2011). Avaliação físico-química de polpas de frutas congeladas comercializadas na região do médio norte matogrossense. Enciclopédia Biosfera, 7(13), 1391-1398.

Pieniz, S., Colpo, E., Oliveira, V. R., Estefanel, V. \& Andreazza, R. Avaliação in vitro do potencial antioxidante de frutas e hortaliças. Ciência e Agrotecnologia, 2009, 33(2), 552-559.

Raimundo, K., Magri, R. S., Simionato, E. M. R. S., \& Sampaio, A. C. (2009). Avaliação física e química da polpa de maracujá congelada comercializada na região de Baurú. Revista Brasileira de Fruticultura, 31(2), 539-543. http://dx.doi.org/10.1590/S0100-29452009000200031

Santos, E. H. F., Neto, A. F., \& Donzeli, V. P. (2016). Aspectos físico-químicos e microbiológicos de polpas de frutas comercializadas em Petrolina (PE) e Juazeiro (BA). Brazilian Journal of Food Technology, 19, e2015089. Recuperado em 02 de junho de 2018, de http://www.scielo.br/pdf/bjtt/v19/1981-6723-bjft-1981-67238915.pdf

Sebastiany, E., Moura, E. R., Rego, E. R., \& Vital, M. J. S. (2009). Perda de vitamina C durante o armazenamento de polpa de acerola congelada. Digital Library of Journal, 27(2), 281-288. http://dx.doi.org/10.5380/cep.v27i2.22039

Silva, B. A., Gordon, A., Jungfer, E., Marx, F., \& Maia, J. G. (2012a). Antioxidant capacity and phenolics of Pouteria macrophylla, an under-utilized fruit from Brazilian Amazon. European Food Research and Technology, 234(5), 761-768. http://dx.doi.org/10.1007/s00217-012-1684-0 
Silva, L. M. R., Maia, G. A., Figueiredo, R. W., Souza, P. H. M., Gonzaga, M. L. C., \& Figueiredo, E. A. T. (2012b). Estudo do comportamento reológico de polpas de caju (Anacardium occidentale, L.), acerola (Malpighia emarginata, D.C.) e manga (Mangifera indica, L.). Revista Semina: Ciências Agrárias, 33(1), 237-248. http://dx.doi.org/10.5433/1679-0359.2012v33n1p237

Silva, N., Junqueira, V. C. A., Silveira, N. F. A., Taniwaki, M. H., Santos, R. F. S., Gomes, R. A. R., \& Okazaki, M. M. (2007). Manual de métodos de análise microbiológica em alimentos (552p.). São Paulo: Blucher.

Singleton, V. L., \& Rossi, J. A. (1965). Colorimetry of total phenolics with phosphomolybdic phosphotungstic acid reagents. American Journal of Enology and Viticulture, 16(3), 144-158.

Urbano, G. R., Maziero, P. P., Kato, T., \& Pedrão, M. R. (2012). Avaliação de parâmetros de qualidade de polpas de acerolas congeladas comercializadas em Londrina - PR. Revista Brasileira de Pesquisa em Alimentos, 3(1), 28-31.

http://dx.doi.org/10.14685/rebrapa.v3i1.65 